\title{
Research on Stress Detection of DC01 Steel via Barkhausen Noise
}

\author{
Xiang Zhang a , Jianping Peng ${ }^{\mathrm{a}, 1}$, Xiaorong Gao ${ }^{\mathrm{a}}$, Jie Bai ${ }^{\mathrm{a}}$ and Jianqiang Guo ${ }^{\mathrm{a}}$ \\ ${ }^{a}$ Photoelectric Engineering Institute, Southwest Jiaotong University, Chengdu, China
}

\begin{abstract}
The industrial component under loading change its mechanical characteristics by stress. It is very important to make clear the distribution of the applied stress in the component to reduce the failure. In this paper, magnetic Barkhausen noise $(\mathrm{MBN})$ method is used to evaluate the stress of DC01 steel. Combined with theory for both magnetic domain and magnetization, this work analyzed MBN signal from energy point of view. Magnetic strength corresponding to the maximum MBN shows a downward trend with the increase of tensile stress. Plots of energy against stress showed a relationship providing a convenient method for detecting stress levels by MBN.
\end{abstract}

Keywords. Magnetic Barkhausen noise, magnetic domain, magnetization

\section{Introduction}

Traditional stress testing methods will cause different damages to the testing materials. Since some components are not allowed to be destroyed, some scholars have proposed a magnetic nondestructive testing method - Barkhausen noise (MBN) to describe the tendency of stress change in materials [1-3]. The Barkhausen effect was found in 1919, while it mainly describes the physical phenomena of a series of electric pulses produced by ferromagnetic materials in an alternating magnetic field [4]. From the microscopic point of view, ferromagnetic materials are composed of domain walls and domains. In the second stage of magnetization, a large number of irreversible emotions are generated in the domain, and MBN signals are generated in the motion between domain walls (Figure 1) [5].

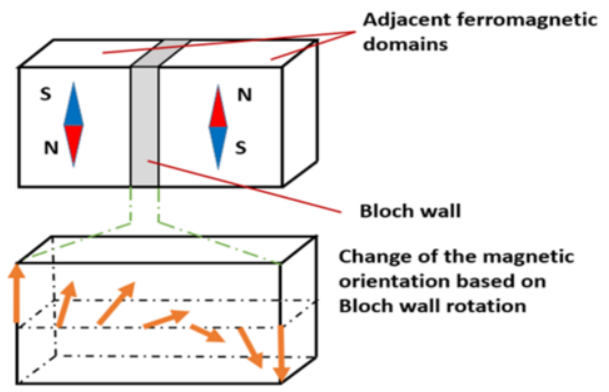

Figure 1. Schematic of domain wall change

\footnotetext{
${ }^{1}$ Corresponding Author, Jianping Peng, Photoelectric Engineering Institute, Southwest Jiaotong University, Chengdu 611756, China; E-mail: peng.jian.ping@126.com.
} 
The method has also been used in many studies for studying the sample's structure. Jiles DC proposed that the strength of the MBN signal is related to the size and distribution of the pinning point of bound domain wall motion in materials [6]. DHAR A pointed out that the external magnetic field will change the distribution of magnetic crystal energy in the domain, breaking the original magneto-static equilibrium state [7]. An external magnetic field may simultaneously affect the internal grains with similar crystal orientation and produce domain wall motion. In the same way, the applied stress breaks the internal crystal structure of the material. The stress effect of single domain crystals was analyzed by Baudouin [8] and Ziman J [9]. Jia L et al. established a correlation model between DW motion and MBN under tensile stress [10]. Also, some scholars extract the peak value, RMS and other characteristics of MBN signal to describe the distributing disciplinarian between MBN and stress [11-13]. J.A. Pérez-Benitéz et al. pointed out that the magnetic strength of peak MBN often appeared near the coercive field [14]. Lo et al. confirmed that the frequency band of MBN signal is almost in the range of $1 \mathrm{kHz}-500 \mathrm{kHz}$ [15]. Leonardo et al. [16] clarify the relationship between the microstructure of steels and magnetic measurements, gives the result with MBN and the hysteresis curve.

In this study, an experimental design with different levels of tensile stress was carried out. Barkhausen noise measurements were carried out from the sample's surface, used for stress profiling. The measured stress profiles were characterized by energy while typical $\mathrm{BN}$ features such as peak and rms value were used to represent the $\mathrm{BN}$ measurements.

\section{Methodology}

The total energy expression in a unit volume of ferromagnetic material is:

$$
E=E_{e x}+E_{k}+E_{\sigma}+E_{d}+E_{H}
$$

In Eq. (1), $E_{e x}$ represents material exchange energy, $E_{k}$ represents material magneto-crystalline anisotropy energy, $E_{\sigma}$ is the magneto-elastic energy, $E_{d}$ represents material demagnetizing energy, and $E_{h}$ represents applied magnetic field energy.

In the absence of a magnetic field, the magneto-static energy of a ferromagnetic body is zero. When atomic spin is not considered, $E_{e x}, E_{d}$, and $E_{h}$ in Eq. (1) do not work, the total energy can be defined as $E_{k}+E_{\sigma}$.

The magneto-crystalline anisotropy energy $E_{k}$ in a cubic crystal can be expressed by the following formula:

$$
E_{k}=K_{1}\left(\alpha_{1}^{2} \alpha_{2}^{2}+\alpha_{2}^{2} \alpha_{3}^{2}+\alpha_{3}^{2} \alpha_{1}^{2}\right)+K_{2} \alpha_{1}^{2} \alpha_{2}^{2} \alpha_{3}^{2}
$$

In Eq. (2), $K_{1}$ and $K_{2}$ are magneto-crystalline anisotropy coupling coefficients $\alpha_{1}$, $\alpha_{2}$, and $\alpha_{3}$ are the cosines of the magnetization vector in three directions of space. 
The applied stress can create regional wall motion without applying a magnetic field. With this condition, the magnetic domain structure in the ferromagnetic crystal will undergo a corresponding deformation (Figure. 2). At this time, in addition to the generalized magneto-crystalline anisotropy energy caused by spontaneous deformation, the external energy of the crystal dominant magnetic stress energy.

Let the external stress tensor expression be:

$\sigma_{i k}=\sigma \gamma_{i} \gamma_{k}$

In Eq. (3), $\gamma_{i}, \gamma_{k}$ is the direction cosine of external stress intensity of $\sigma$.

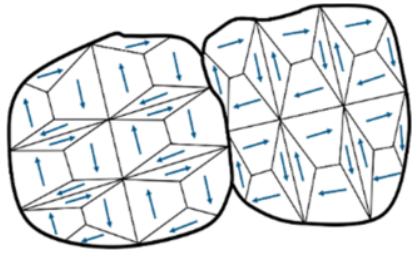

(a) Primitive morphology of crystals

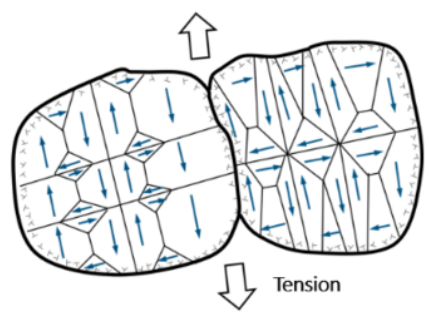

(b) Domain after Loading Stress

Figure 2. Comparison of Domain Structures before and after Stress Loading

An applied magnetic field may simultaneously affect internal grains with similar crystal orientation and generate domain wall motion. In the elastically deformed region, constant stress can be expressed as an additional magnetic field $H_{\sigma}$, which is a function of magnetoelastic energy $E_{\sigma}$ and magnetization $M$, thereby:

$$
H_{\sigma}=\frac{1}{\mu_{0}} \frac{\partial E_{\sigma}}{\partial M}
$$

In Eq. (4), where $\mu_{0}$ is the vacuum permeability, assuming that there is no transverse strain, the magnetostriction is isotropic, the form of the magnetoelastic energy generated by the stress can be simplified as:

$$
E_{\sigma}=-\frac{3}{2} \lambda \sigma \cos ^{2} \vartheta
$$

In Eq. (5), $\lambda$ is the amount of magnetostriction, and $\vartheta$ is the angle of applied stress and magnetization. When the magnetic field has the same direction with stress, Eq. (4) can be expressed as:

$$
H_{\sigma}=\frac{3 b \sigma M}{\mu_{0}}
$$


Where in Eq. (6), $M$ is the magnetization, $b$ is the second-order magneto-strictive coefficient, $\mu_{0}$ represent the vacuum permeability, $\sigma$ is the strength of applied stress.

$s(t)$ is the original signal, $s_{J}^{1}, s_{J}^{2}, \ldots, s_{J}^{i}$ is the $J^{\text {th }}$ layer decomposition component of the signal, and $E_{J}^{1}, E_{J}^{2}, \ldots, E_{J}^{i}$ is the energy of the $J^{t h}$ layer wavelet decomposition amount respectively. Then the energy of each wavelet decomposition can be expressed as:

$$
E_{J}^{i}(t)=\sum_{\tau=t_{0}}^{t}\left(s_{J}^{i}(\tau)\right)^{2}
$$

The overall energy of the signal can be expressed as:

$$
E_{\text {total }}(t)=\sum_{J} E_{J}^{i}(t)
$$

\section{Experimental Procedure}

DC01 steel is widely used in manufacturing of high-performance and critical components. Both chemical composition and mechanical parameter are given in Table 1 and Table 2, respectively. Non-oriented magnetic steel sheets, DC01, with the 40-mm width $\times 310$-mm length $\times 1.0$-mm thickness were used for the specimens, as shown in Figure 3. During tensile tests, stresses of various amplitudes within the elastic limit were applied using a mechanical testing system (see Figure 4.). MBN measurements were taken for a monotonically increasing load with increments of $16.6 \mathrm{MPa}$.

The measurement was made by using a sinusoidal magnetic wave of $1 \mathrm{~Hz}$ with $12 \mathrm{~V}$ applied field. The MBN signal was obtained by a pick-up coil after superimposed 10 times and then amplified by an amplifier with a gain of $20 \mathrm{~dB}$.

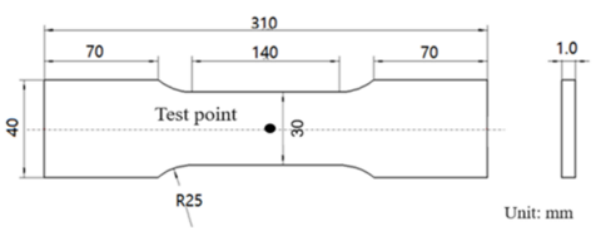

Figure 3. Dimension diagram of DC01 Steel.

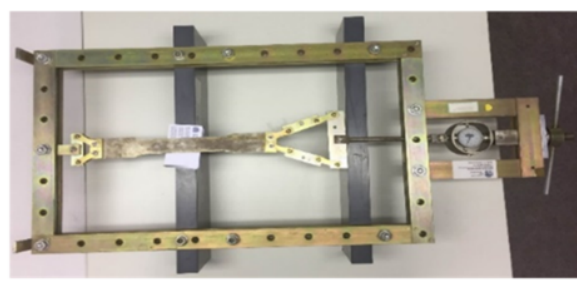

Figure 4. Mechanical testing system.

Table 1. Chemical composition \% (mass fraction) of the DC01 steel

\begin{tabular}{rccccc}
\hline Sample name & Standard & C & Mn & P & S \\
\hline DC01 & EN10130:2006 & $\leq 0.12$ & $\leq 0.60$ & $\leq 0.045$ & $\leq 0.045$ \\
\hline
\end{tabular}


Table 2. Mechanical parameter of the DC01 steel

\begin{tabular}{ccrc}
\hline Sample name & $\begin{array}{c}\text { Yield strength } \\
\text { No more than }\end{array}$ & Tensile strength & $\begin{array}{c}\text { Elongation after breaking } \\
\text { No less than }\end{array}$ \\
\hline DC01 & $280 \mathrm{MPa}$ & $270-410 \mathrm{MPa}$ & $28 \%$ \\
\hline
\end{tabular}

The sampling frequency was equal to $100 \mathrm{kHz}$, conventional signal processing technology based on the Fourier spectrum helps analyze the frequency characteristics. Set the signal divided into eight parts by three-layer wavelet packet decomposition. Through the energy distribution, the energy inside the domain at each stage can be evaluated.

\section{Results}

The Barkhausen noise measurements were carried out from the DC01 steel after applying a constant loading. The behavior of the Peak and Root Mean Square (RMS) value on samples can be seen in Figure 5. and Figure 6., respectively. It is shown that both Peak and RMS amplitude of MBN was found to correlate with applied stress, showing a clear rising trend for tensile stress under yield strength. It is noteworthy that RMS has a higher coefficient $r^{2}=0.9542$ compared with the Peak coefficient $r^{2}=0.9127$, which means a better correlation can be found with RMS condition.

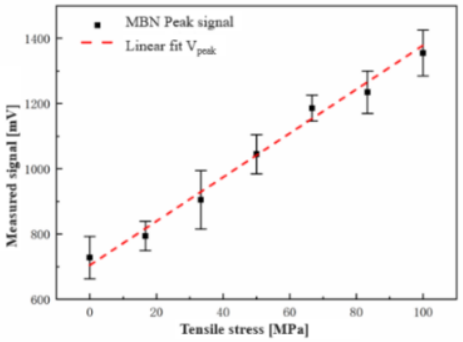

Figure 5. MBN peak amplitude as a function of applied stress.

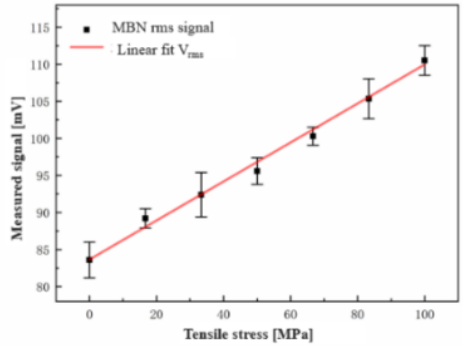

Figure 6. $M B N$ rms amplitude as a function of applied stress.

To further evaluate the effectiveness of the magnetic techniques in determining tensile stresses, energy distribution with different stress levels is presented in Figure 7. It was shown that MBN signal mainly distributes in low-frequency region. Moreover, the energy of each wavelet decomposition shows sensitivity to applied tensile stress, especially at the low-frequency part. Consider using energy to express MBN distribution, Figure 8. presents the distribution between energy and magnetic strength against stress. Increasing stress also increases total energy, but magnetic strength of maximum MBN showing a downward trend. With tensile stress's influences, the shape of hysteresis loop becomes narrower. Therefore, magnetic strength of Peak value decrease. 


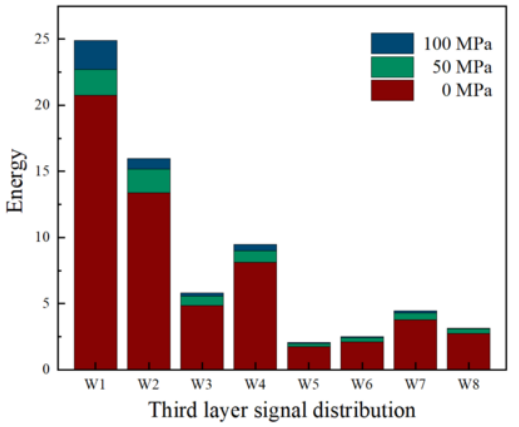

Figure 7. Energy Distribution.

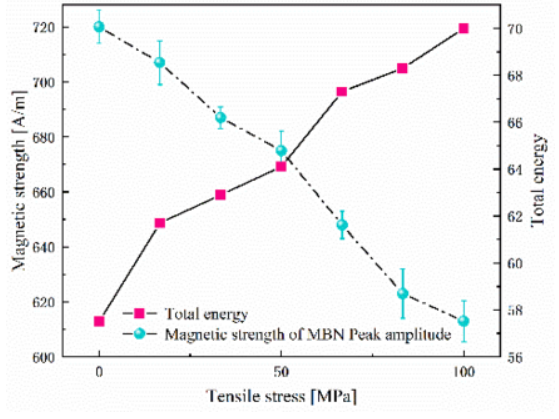

Figure 8. Superposition of total energy and magnetic strength for DC01 steel.

\section{Conclusion}

This paper describes a method for detecting stress in the surface of magnetic materials based on the measurement of MBN. Both peak and rms amplitude of the MBN has been experimentally shown a vary linearly with stress. Therefore, this result provides a useful calibration curve for determining stress. Then energy was used to evaluate the effectiveness of the MBN. A relationship was found between energy and parabola peak position (magnetic strength), which is associated with the shape of hysteresis loop.

\section{Acknowledgement}

This work is supported by National Nature Science Foundation of China (Grant No. 61771409), and the authors wish to acknowledge them for their support.

\section{References}

[1] Samimi A A, Krause T W, Clapham L. Stress Response of Magnetic Barkhausen Noise in Submarine Hull Steel: A Comparative Study[J]. Journal of Nondestructive Evaluation, 2016, 35(2):32.

[2] Kasai N, Koshino H, Sekine K, et al. Study on the Effect of Elastic Stress and Microstructure of Low Carbon Steels on Barkhausen Noise[J]. Journal of Nondestructive Evaluation, 2013, 32(3):277-285.

[3] Tsuchida Y, Enokizono M. Residual stress evaluation by Barkhausen signals with a magnetic field sensor for high efficiency electrical motors[J]. Aip Advances, 2018, 8(4):047608.

[4] Barkhausen H. Two phenomena revealed with the help of new amplifiers[J]. Phys, 1919,20:1-3.

[5] Mierczak L, Jiles D C, Fantoni G. A New Method for Evaluation of Mechanical Stress Using the Reciprocal Amplitude of Magnetic Barkhausen Noise[J]. IEEE Transactions on Magnetics, 2011, 47(2):459-465.

[6] Jiles DC. Dynamics of domain magnetization and the Barkhausen effect[J]. Czechoslovak Journal of Physics, 2000, 50(8):893-924.

[7] Dhar A, Clapham L, Atherton D L. Influence of uniaxial plastic deformation on magnetic Barkhausen noise in steel[J]. NDT\&E International, 2001, 34(8):507-514.

[8] Baudouin J B, Monnet G, Perez M, et al. Effect of the applied stress and the friction stress on the dislocation dissociation in face centered cubic metals[J]. Materials Letters, 2013, 97(15):93-96.

[9] Ziman J, Kladivová M, Zagyi B. Influence of a "domain-drag effect" on the movement of a single boundary between circular domains in stress-annealed amorphous CoFeSiB wire[J]. Journal of Magnetism \& Magnetic Materials, 2003, 254(254):176-178. 
[10] Liu J, Tian G Y, Gao B, et al. Micro-macro characteristics between domain wall motion and magnetic Barkhausen noise under tensile stress[J]. Journal of Magnetism and Magnetic Materials, 2020, 493 : 165719.

[11] Ktena A, Hristoforou E, Gerhardt G J L, et al. Barkhausen noise as a microstructure characterization tool[J]. Physica B: Condensed Matter, 2014, 435:109-112.

[12] Sorsa A, Kauko Leiviskä, Santaaho S, et al. Quantitative prediction of residual stress and hardness in case-hardened steel based on the Barkhausen noise measurement[J]. NDT\&E International, 2012, 46(1):100-106.

[13] Kypris O. Detection of sub-surface stresses in ferromagnetic materials using a new barkhausen noise method[D]. Ames: Iowa State University, 2015.

[14] J.A.Pérez-Benitéz, Hernandéz-Espina, Ortiz-Martinéz, Unwrapping the influence of multiple parameters on the magnetic Barkhausen noise signal using self-organizing maps[J]. NDT \& E International, 2013, 54:166-170

[15] Lo, C.C.H, Jakubovics J P. Monitoring the microstructure of pearlitic steels by magnetoacoustic emission[J]. Journal of Applied Physics, 1997, 81(8):4069-4071.

[16] Leonardo F. T. Costa, Marcos F. de Campos et al. Hysteresis and Magnetic Barkhausen Noise for SAE 1020 and 1045 Steels With Different Microstructures [J]. IEEE Transactions on Magnetics, 2014. 